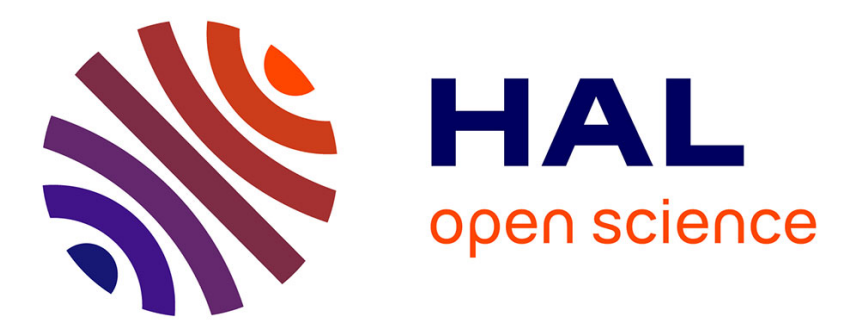

\title{
Les trajets domicile-école des élèves de primaire peuvent-ils constituer un point de départ pour construire des apprentissages spatiaux ?
}

\author{
Elsa Filâtre
}

\section{- To cite this version:}

Elsa Filâtre. Les trajets domicile-école des élèves de primaire peuvent-ils constituer un point de départ pour construire des apprentissages spatiaux ?. Géocarrefour - Revue de géographie de Lyon, 2020, 94 (2), 10.4000/geocarrefour.14769 . hal-02932327

\section{HAL Id: hal-02932327 https://hal.science/hal-02932327}

Submitted on 26 Apr 2021

HAL is a multi-disciplinary open access archive for the deposit and dissemination of scientific research documents, whether they are published or not. The documents may come from teaching and research institutions in France or abroad, or from public or private research centers.
L'archive ouverte pluridisciplinaire HAL, est destinée au dépôt et à la diffusion de documents scientifiques de niveau recherche, publiés ou non, émanant des établissements d'enseignement et de recherche français ou étrangers, des laboratoires publics ou privés. 


\section{Géocarrefour}

$94 / 2 \mid 2020$

Des lieux pour apprendre et des espaces à vivre :

l'école et ses périphéries. Les dehors et les ailleurs

\section{Les trajets domicile-école des élèves de primaire peuvent-ils constituer un point de départ pour construire des apprentissages spatiaux?}

Can the home to school journeys of primary school children constitute a starting point for building spatial learning?

\section{Elsa Filâtre}

\section{(2) OpenEdition}

\section{Journals}

Édition électronique

URL : http://journals.openedition.org/geocarrefour/14769

DOI : 10.4000/geocarrefour.14769

ISSN : 1960-601X

Éditeur

Association des amis de la Revue de géographie de Lyon

Ce document vous est offert par Université Toulouse 2 - Jean Jaurès

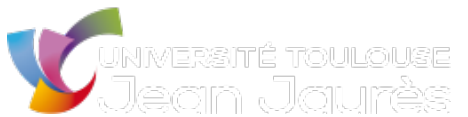

Référence électronique

Elsa Filâtre, "Les trajets domicile-école des élèves de primaire peuvent-ils constituer un point de départ pour construire des apprentissages spatiaux ? », Géocarrefour [En ligne], 94/2 | 2020, mis en ligne le 17 avril 2020, consulté le 26 avril 2021. URL : http://journals.openedition.org/geocarrefour/ 14769 ; DOI : https://doi.org/10.4000/geocarrefour.14769

Ce document a été généré automatiquement le 26 avril 2021

(c) Géocarrefour 


\section{Les trajets domicile-école des élèves de primaire peuvent-ils constituer un point de départ pour construire des apprentissages spatiaux?}

Can the home to school journeys of primary school children constitute a starting point for building spatial learning?

Elsa Filâtre

\section{Introduction}

1 Que savent les enseignants des pratiques spatiales de leurs élèves? En quoi ce questionnement peut-il les aider à investir davantage l'exploration de l'environnement proche des élèves en classe de géographie? Cet article propose d'envisager le trajet domicile-école au sein des travaux de didactique de la géographie afin d'explorer les enjeux de la prise en compte de la spatialité des élèves dans différents types de territoires. La mobilité quotidienne fait partie des expériences spatiales des élèves et elle a fait l'objet de nombreux travaux de recherche francophones et anglo-saxons dans des perspectives très différentes. Elle a pu être étudiée selon des cadres sociologiques et/ou géographiques d'analyse d'inégalités sociales, territoriales ou genrées; à l'occasion d'étude des effets de pratiques sociales ou publiques ou encore dans une problématique d'éducation à la santé. Le recueil de données de cet article est situé dans le cadre d'une recherche didactique plus large ${ }^{1}$ sur les effets de l'enseignement à partir de l'environnement proche des élèves, en géographie en classe de CM1 et il devenu le point d'appui d'une recherche exploratoire sur les pratiques de déplacements des élèves sur le trajet domicile-école en termes d'autonomie, de temps et de moyen de transport. En interrogeant des élèves de 8 à 11 ans issus de trois types d'espaces différents (urbain, périurbain et rural) sur les modalités de leur mobilité quotidienne, il est possible de questionner leur degré d'autonomie ainsi que l'influence des modes de 
vie liés à ce type de territoires sur les conditions de ces déplacements. Dans une perspective didactique, l'objectif est, d'une part, de caractériser le trajet domicile-école comme une expérience spatiale à même de construire des compétences de spatialité, puis de l'interroger dans le cadre de l'étude des mobilités enfantines afin d'en comprendre les enjeux et enfin de se demander comment l'expérience spatiale des enfants peut être prise en compte dans l'enseignement de la géographie.

\section{Cadre théorique : Les trajets domicile-école, un espace-temps particulier}

\section{Le trajet domicile-école, une expérience de spatialité}

2 S'intéresser à la mobilité quotidienne des enfants suppose d'identifier en quoi elle constitue une expérience spatiale. Le renouvellement épistémologique de la géographie a fait de la spatialité un concept central qui met en jeu les manières dont l'individu est confronté à l'espace. Ainsi, au-delà de la simple localisation d'un phénomène, ou encore de l'observation d'un aspect d'une réalité sociale, la spatialité entretient un rapport systémique avec l'espace qui en devient l'environnement (Lussault, 2013). Elle est identifiée comme " une expérience individuelle et sociale qui engage la personne, son corps, ses capacités, ses sens et la met à l'épreuve de l'espace (...).» (Lussault, 2015, p. 409). Le tournant géographique des années 1990 situe la spatialité dans les modes d'habiter en prenant en compte, au-delà d'un rapport à l'espace, les interrelations humaines. On considère alors, avec les travaux de Mathis Stock, qu'habiter c'est « faire avec l'espace » (2015), et parce que l'espace met en jeu la question de la distance et confronte les individus et les groupes à l'état séparatif, le concept d'habiter forme « la spatialité typique des individus et des groupes » (Lussault, 2013, p. 905). Si les travaux d'Armand Frémont sur l'espace vécu (Frémont, 1976) ou d'Antoine Bailly sur les représentations (Bailly, 1977 ; 1989) ont placé le concept d'expérience dans le champ de la géographie, l'étude des spatialités, l'art de faire avec l'espace permet d'y intégrer l'engagement de l'individu, la dimension interactionnelle et le renforcement des compétences spatiales (Lussault, 2015). Le trajet domicile-école des élèves de primaire peut être ainsi considéré comme une expérience spatiale, en tant qu'espace-temps spécifique, qui engage l'individu dans une pratique spatiale, faite de distance, de lieux, de limites, de franchissements. Ainsi, c'est une expérience qui mobilise un grand nombre de compétences spatiales. Thierry Ramadier, dans le cadre de l'étude des liens entre mobilité quotidienne et attachement au quartier (Ramadier, 2007), situe le trajet domicile-école dans le champ des expériences spatiales qui permettent la construction d'une représentation cognitive de l'espace. Ainsi, selon lui, les «difficultés à se représenter l'espace ne permettent à un individu ni de diversifier les espaces fréquentés ni de construire en retour une représentation cognitive de l'espace qui soit étendue et structurée» (2007, p. 132). Dans l'article consacré aux compétences de spatialité, Michel Lussault explique que toute action spatiale met en jeu des compétences (Lussault, 2013). Si on considère que le déplacement d'un enfant, de son domicile à son école, est une expérience spatiale, on peut alors établir que cette expérience est à même d'en structurer une grande diversité : des compétences de parcours qui permettent à l'individu de composer et d'assurer un itinéraire; des compétences métriques dans le sens où le déplacement permet de maîtriser et de 
mesurer les distances en prenant conscience du proche et du lointain ; mais également des compétences de découpage qui permettent d'identifier et de délimiter des unités spatiales. Ainsi, si le trajet domicile-école peut être considéré comme une expérience de spatialité permettant la construction, l'appropriation et la mobilisation de compétences spatiales essentielles, il s'agit à présent de le situer dans les expériences de mobilité des enfants.

\section{La place du trajet domicile-école dans la mobilité des enfants}

3 La mobilité des enfants a fait l'objet de plusieurs travaux, d'abord au sein du monde anglophone dans lequel l'étude de Sanford Gaster fait référence (Gaster, 1991). En effet, dans les années 1990, il a fait une recherche sur la place des enfants dans l'environnement public entre 1915 et 1976 à New York. Il s'est appuyé sur des entretiens réalisés auprès de 39 adultes de différentes générations pour déterminer leur expérience spatiale en tant qu'enfant. Ces entretiens ont été croisés avec des rapports de recensement sur les changements démographiques. La recherche montre que, depuis les années 1940, la place des enfants dans l'environnement public et, en particulier, dans leur quartier, a décliné. Ainsi, l'âge des premières sorties sans surveillance a été repoussé, le nombre de lieux visités par les enfants a diminué et a souvent été réduit à l'environnement immédiat du domicile, les restrictions imposées par les parents se sont renforcées et les activités sont devenues de plus en plus supervisées par des professionnels. En France, les travaux de Sandrine Depeau et Thierry Ramadier (Depeau, 2013; Depeau et Ramadier, 2005) et ceux de Florence Huguenin-Richard, qui a travaillé sur l'impact des zones 30 (zone de limitation de vitesse à $30 \mathrm{~km} /$ heure) sur la mobilité des enfants (2010), ont pu mettre en avant les mêmes constats. Cette dernière explique que, dans le domaine de l'aménagement et de l'urbanisme, la place de l'enfant dans la ville est souvent réduite à la mobilité scolaire sous un angle avant tout sécuritaire. Dans leurs travaux, Sandrine Depeau et Thierry Ramadier constatent que la réduction de la mobilité des enfants a des conséquences multiples, à la fois sur le développement de l'autonomie des enfants, puisque leurs expériences spatiales sont fortement limitées, mais également sur leur dépendance à l'adulte (Depeau et Ramadier, 2005). Sandrine Depeau explique que les enfants entrent dans la mobilité par l'immobilité. Elle a ainsi étudié les pratiques de pédibus pour effectuer les trajets domicile-école chez des élèves de primaire dans l'agglomération rennaise et a pu montrer la place importance des choix parentaux dans la mobilité des enfants qui, en tant que valeurs et normes, conduisent à réduire l'autonomie des enfants dans leur mobilité (Depeau, 2013). Vincent Kaufmann et Éric Widmer (2005), ont exploré les liens entre la mobilité et le fonctionnement des familles dans les espaces urbains et périurbains. Ainsi, l'habitat périurbain est un choix porteur pour les familles parce qu'il participe fortement, par la présence d'un jardin attenant au domicile, au développement $\mathrm{du}$ jeune enfant, mais par ailleurs il implique des pratiques d'accompagnement en raison du manque d'aménités disponibles. Le contexte urbain mobilise plutôt des restrictions de mobilité quotidienne avant tout pour des raisons sécuritaires largement associées aux dangers que représente la fréquentation de la rue. Il en résulte que ces contextes urbain et périurbain ont pour conséquence un déplacement des étapes d'acquisition de la mobilité autonome de l'enfance vers l'adolescence. En effet, la fréquentation de la rue par les enfants décline au profit d'un mouvement d'intériorisation centré sur le domicile et la chambre, en particulier dans 
les espaces périurbains bénéficiant d'une augmentation de la surface habitable (Didier Fèvre, 2014). Les enfants ont moins souvent le droit d'aller seul à l'école ou de jouer dans la rue sans la surveillance d'un adulte et perdent ainsi l'acquisition progressive d'une mobilité autonome au détriment d'une construction plus rapide au moment de l'adolescence vers 13-14 ans. Les auteurs concluent sur l'hypothèse d'un lien fort entre la croissance de l'étalement urbain et les fonctionnements familiaux « fusionnels et fermés». Ce déplacement des pratiques de mobilité de l'enfance vers l'adolescence a été confirmé par les travaux de Devaux, Oppenchaim et Proulhac (2016), dans une étude diachronique observant vingt ans de pratiques de mobilité des adolescents d'île-deFrance de 11 à 14 ans. Pour les auteurs, la mobilité autonome est une composante essentielle de l'habiter car elle permet «de s'affranchir de la tutelle parentale et d'apprendre à trouver sa place dans l'espace public ", elle est à la base « de dispositions, de manières d'habiter qui structureront en partie leur vie future et leur insertion professionnelle (...)» (op. cit., p. 1-2).

En France, assez peu de travaux sur la mobilité enfantine traitent spécifiquement du trajet domicile-école des enfants, mais au Québec et aux États-Unis plusieurs recherches ont apporté des résultats intéressants pour constater le déclin de l'autonomie des enfants. Tracy Mac Millan (McMillan, 2005) a pu mettre au jour les facteurs qui influencent le choix du mode de transport du trajet domicile-école aux États-Unis pour évaluer la place du transport actif (piéton ou cycliste) dans une perspective d'éducation à la santé. Elle mobilise différents facteurs : ceux liés à la sécurité du quartier (réelle ou perçue), les normes socio-culturelles et les attitudes parentales, mais également la forme urbaine. Elle a constaté que l'augmentation de la longueur des blocs, la largeur des rues et la diminution de la présence de trottoirs dans les rues des collectivités ont entraîné une diminution de la marche à pied et de l'activité physique. L'ensemble de ces facteurs influe sur les décisions parentales par rapport à la mobilité quotidienne de leurs enfants. Par ailleurs, elle explique que le trajet domicile-école est souvent considérée comme un évènement non pas isolé et donc réfléchi en tant que tel, mais lié à une mobilité plus large, souvent en relation avec les déplacements vers le lieu de travail des parents dans une perspective de maximisation de l'utilité d'un déplacement. Paul Lewis et Juan Torres (Lewis et Torres, 2010) se sont appuyés sur ces travaux pour étudier les modes de déplacement des enfants de plusieurs écoles de Montréal et de Trois Rivières afin de comprendre les raisons du déclin du transport actif et de la mobilité autonome des enfants sur le trajet domicile-école. Ils ont ainsi pu montrer que l'autonomie des enfants dépendait de différents facteurs : la forme urbaine qui a une influence sur le choix du transport actif au regard de la distance qu'elle crée entre l'école et le domicile, la gestion scolaire des familles (détournement de la carte scolaire, projet éducatif des communes) et le rapport parents-enfants (par exemple pour la primauté donnée à la sécurité sur la mobilité autonome). Ils ont ainsi pu montrer que le trajet scolaire était l'expression d'un rapport à la ville et que le facteur déterminant de la construction de la mobilité de l'enfant était avant tout lié à la pratique de mobilité des parents. En effet, ceux-ci jouent un rôle fondamental en termes de choix d'école ou de quartier, de modèle de mobilité (transport actif ou motorisation) et de préoccupation sécuritaire. Le renforcement de la protection des enfants par la surveillance de leur mobilité a été étudié par Trine Fotel et Thyra Thomsen (Fotel et Thomsen, 2003) qui ont montré que les pratiques d'accompagnement motorisé des parents étaient également un moyen utilisé par les parents pour surveiller leurs enfants. Ce constat a amené Paul Lewis et 
Juan Torres à s'interroger sur la place des enfants dans la ville, en montrant notamment que la prise en compte des enfants en tant qu'acteurs de leur propre mobilité les rendrait capable de construire leur propre sécurité par leur occupation de l'espace. Ainsi, les recherches sur les mobilités enfantines et en particulier sur la mobilité quotidienne concluent à un déclin de la mobilité autonome des enfants, propre à questionner la construction de leur spatialité et donc de leur habiter. Olivier Lazzarrotti (2014) donne ainsi trois dimensions à l'habiter, une manière d'être au monde, dans la lignée des travaux phénoménologiques, l'étude des pratiques en considérant qu'habiter c'est faire avec l'espace et enfin la notion de co-habitation, dans le sens où habiter est une expérience de soi et des autres à travers le monde. La mobilité quotidienne place ainsi l'enfant dans un rapport à l'espace, aux lieux de son quotidien et à la circulation entre ces lieux et le trajet domicile-école peut être conçu comme une expérience de l'habiter en tant que pratique spatiale.

\section{La prise en compte de l'expérience spatiale des enfants dans l'enseignement de la géographie}

5 La prise en compte de l'expérience spatiale des élèves est un enjeu récent des programmes de géographie et des travaux des didacticiens.

6 Le programme de $2016^{2}$ articule de manière intrinsèque la spatialité des individus et des groupes sociaux, à une échelle locale, avec les pratiques sociales qu'ils exercent au quotidien : « habiter, se loger, travailler, avoir des loisirs, se cultiver, se déplacer », en plaçant la notion d'habiter au centre des concepts à construire avec les élèves. La place de l'élève y est déterminante, puisqu'il est proposé de s'appuyer sur « un travail préliminaire sur les représentations des élèves (...) pour montrer comment chacun habite et s'approprie son espace proche $»^{3}$. La notion même d'habiter est devenue l'architecture des programmes de géographie de cycle 3. Michel Lussault, qui était président du Conseil Supérieur des Programmes lors de leur construction, conçoit l'habiter "comme ce qui consiste à «prendre place» dans et par l'expérience et « naître au monde continûment (...) à travers cette expérience » (Lussault, 2015). Ainsi l'école participe à la construction de la spatialité en étant à la fois instituante et en même temps lieu de construction d'expériences spatiales. En partant de l'expérience que les élèves ont d'un trajet quotidien, celui qu'ils font pour aller à l'école, on peut imaginer s'appuyer sur leur expérience, à la fois singulière et collective, pour construire des compétences spatiales.

7 Prendre en compte l'expérience spatiale des élèves est un des enjeux récents des travaux des didacticiens et suppose de s'interroger sur la place de l'échelle locale dans la construction d'apprentissages géographiques et la place de la prise en compte de l'expérience des élèves dans la mise en œuvre de situation d'enseignementapprentissage à l'école. Comme l'ont montré Christine Vergnolle-Mainar, Sophie Gaujal et Caroline Leininger-Frézal (2017), le local a eu une place variable dans l'enseignement de la géographie et les programmes lui ont donné différentes fonctions: se repérer dans l'espace de la classe, de l'école et du quartier et le représenter; construire une première culture géographique en procédant par une décentration progressive des espaces proches aux plus lointains, mais également permettre aux élèves d'être des producteurs de savoirs géographiques en les mettant en situation d'investigation sur l'espace environnant l'école par exemple. Sylvie Considère, Christophe Duhaut et 
Nicolas Lebrun (2009), ont montré, en s'appuyant sur les programmes de cycle 2 et 3 de 2008, que le quartier est plutôt envisagé en tant que support de représentation de l'espace au cycle 2, comme un élément de l'espace urbain dans les classes du cours moyen du cycle 3, sans forcément qu'il soit lié à la proximité des élèves, et la plupart du temps comme une propédeutique à la découverte du monde. Plusieurs travaux de didacticiens ont pris en compte l'expérience spatiale des élèves dans le cadre d'une articulation entre géographie spontanée et géographie expérientielle. Sophie Gaujal, dans sa thèse (2016), propose une formalisation de la distinction entre une géographie spontanée et une géographie raisonnée. En s'appuyant sur les travaux de Denis Retaillé (1997) et de Jean-François Thémines (2006), elle définit la géographie spontanée comme une géographie élaborée par les individus de manière personnelle en s'appuyant sur leur propre pratique de l'espace et en particulier leur mobilité quotidienne, tandis que la géographie raisonnée correspond, elle, a une géographie élaborée, qui s'appuie sur un ensemble d'énoncés, de notions et de concepts et qui vise la théorisation. On peut donc situer l'appui sur la mobilité quotidienne des élèves dans le champ de la géographie spontanée. Caroline Leininger-Frézal (Leininger-Frezal, 2018), quant à elle, définit la géographie expérientielle en s'appuyant sur deux références, celle de l'apprentissage expérientiel théorisé par David Kolb (Kolb, 2014) qui favorise un apprentissage actif plaçant l'apprenant au cœur du processus d'apprentissage dans un processus réflexif, mais elle s'insère également dans le champ de la géographie herméneutique (Thémines, 2007 ; Retaillé, 1997) ou la géographie des processus (Hertig, 2009) qui supposent de prendre en compte les acteurs, leurs représentations et leurs pratiques pour construire une pensée spatiale. En s'appuyant sur les représentations initiales des élèves et leurs pratiques de l'espace comme point de départ d'une situation d'apprentissage, la géographie expérientielle vise à construire des analyses de l'espace perçu ou vécu par les élèves. Ainsi, les expériences spatiales des élèves et en particulier celles liées à la mobilité quotidienne représentent un point d'appui pertinent pour développer des apprentissages géographiques, à condition de s'interroger sur la réalité de ces pratiques.

\section{Une étude des trajets domicile-école d'élèves d'école situées dans des territoires différents}

\section{Présentation du corpus de données : description du questionnaire, de la passation et de l'analyse}

8 L'objectif du questionnaire n'était pas d'interroger les élèves sur leurs pratiques de mobilité quotidienne, mais plutôt de vérifier si l'expérience spatiale que les élèves avaient vécue lors de la mise en œuvre d'une ingénierie didactique au cours du trimestre pouvait expliquer les progrès constatés chez une grande majorité d'élèves dans leur manière de représenter l'espace proche de l'école ${ }^{4}$. Autrement dit, il s'agissait d'écarter un biais d'interprétation des résultats qu'aurait peut-être pu expliquer la mise en place d'une pratique autonome du trajet domicile-école depuis leur entrée en CM1. En effet, si les élèves avaient développé une pratique autonome du trajet domicile-école en même temps qu'en classe ils construisaient des compétences spatiales à partir de l'espace proche de l'école, il aurait pu être beaucoup plus délicat d'attribuer les progrès réalisés à l'ingénierie didactique. 
9 La mobilité quotidienne des élèves a été interrogée au moyen d'un questionnaire présenté à six classes d'élèves de CM1, CE2-CM1 et CM1-CM2 de cinq écoles différentes en Haute-Garonne soit cent quarante et un élèves. Les questions portaient sur leurs pratiques du trajet domicile-école en termes d'autonomie, de temps et de modalités (fig.1). Les cinq questions proposées ont donné lieu à un traitement uniquement quantitatif, puisque l'objectif était de repérer l'autonomie des élèves sur le trajet.

Figure 1 : Questionnaire passé aux élèves $(N=141)$

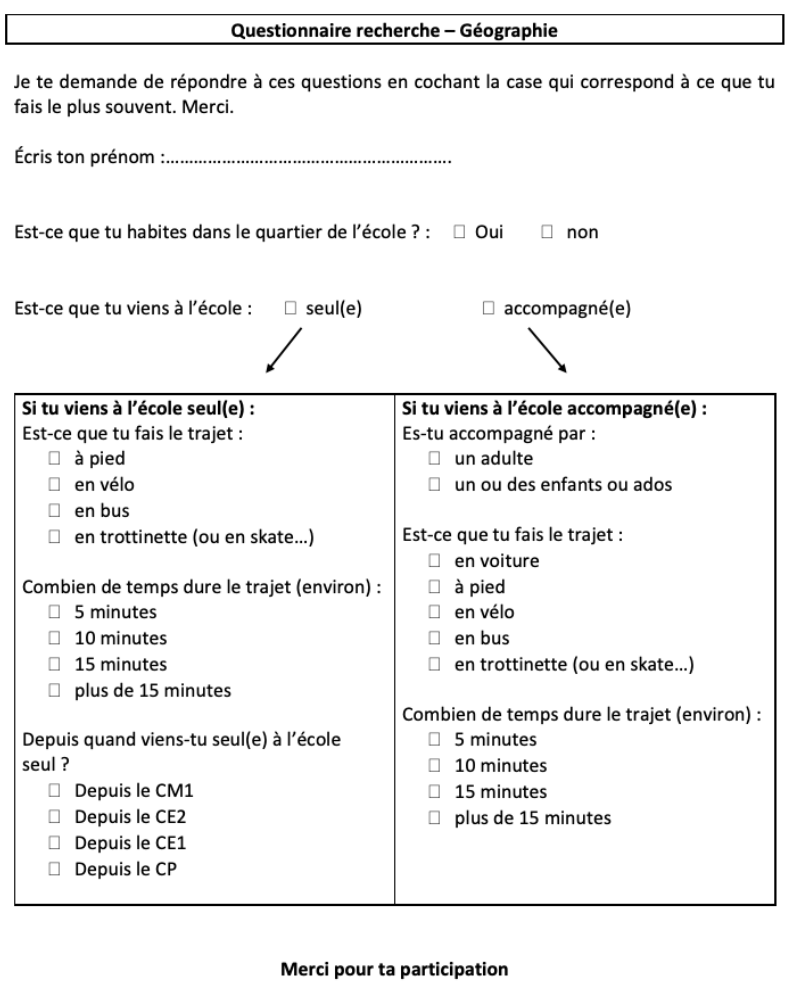

10 En ce qui concerne la passation, elle a été réalisée en présence de la chercheuse, ce qui a pu permettre de lever un certain nombre de difficultés. Ainsi, les élèves étaient invités à chaque fois à ne cocher qu'une seule réponse, celle correspondant à la pratique réalisée le plus souvent. Pour le cas des enfants placés en garde alternée, il était demandé qu'ils remplissent le questionnaire en s'appuyant sur le domicile du secteur de l'école ou celui correspondant à la semaine en cours si les deux domiciles étaient sectorisés sur l'école.

Le corpus a été analysé à partir d'un tri à plat.

\section{Description du corpus : les territoires}

12 Les écoles concernées relèvent de territoires différents : une classe correspond à un territoire rural, deux classes à un espace périurbain, et trois classes relèvent de trois territoires différents d'une même métropole: le centre-ville, ce que l'on pourrait appeler un péricentre et un quartier situé à la périphérie de la ville. Cette caractérisation des espaces des écoles donne lieu à une typologie identifiée et nommée dans les résultats comme suit: centre-ville, péricentre, périphérie de la ville, périurbain 1 et 2 et rural. 
13 L'échelle concernée par le travail réalisé avec les élèves s'apparente à l'aire de ce que l'on peut appeler quartier dans le cadre d'une échelle locale d'observation de l'environnement proche de l'élève. C'est le terme qui a été choisi avec les classes. Afin de mieux cerner les territoires qui ont été interrogés, il peut être utile de les décrire. Pour ce faire, plusieurs caractéristiques ont été relevées qui proviennent de trois types de sources différentes : les dossiers complets des communes de l'INSEE, les observations de différentes couches et données du Géoportail et les observations directes sur le terrain. Les données statistiques proviennent des « Dossiers communes» de l'échelon « commune » du site de l'INSEE ${ }^{5}$ qui établissent un ensemble d'indicateurs sous la forme de graphiques et de tableaux classés par thème (évolution et structure de la population, caractéristiques du logement, de l'emploi...). On y a retenu les catégories et types de logements (Série LOG T2) pour y repérer la place des maisons individuelles et des appartements, le lieu de travail des actifs de 15 ans ou plus ayant un emploi dans la commune de résidence (ACT T4) qui permet de repérer si la majorité de la population travaille dans la commune de résidence et enfin la part des moyens de transports utilisés pour se rendre au travail (ACT G2) pour le comparer aux résultats obtenus sur le corpus étudié. Les observations directes ont été réalisées lors de la mise en place de l'ingénierie didactique par l'observation de l'environnement proche de l'école: forme des rues et des routes, aménités, formes de l'habitat, types de commerces ou de services..., ces observations directes ont été vérifiées et complétées par l'observation de plusieurs couches de données du site Géoportail de l'IGN (Institut Géographique National) ${ }^{6}$ : les différents fonds de carte, les photographies aériennes, les couches historiques par exemple.

14 Les espaces concernés sont très différents par leur physionomie. L'école appelée « Rural » dans la typologie est une école ancienne située en proximité immédiate de la mairie et de l'église qui forme le centre d'un bourg de 910 habitants situé à $63 \mathrm{~km}$ de la grande métropole du département à proximité immédiate d'un axe autoroutier structurant. Le rapport sur la commune de l'INSEE ${ }^{7}$ confirme une forme d'habitat en maisons individuelles $(87,7 \%$ des habitants de la commune résident en maisons individuelles selon les chiffres de 2015) et un nombre important d'actifs qui ne travaillent pas dans la commune puisque $76,7 \%$ d'entre eux travaillent dans une autre commune que celle de résidence en 2015 , ce qui la situe en rattachement à l'aire urbaine métropolitaine avec un mode de vie qui se rapproche du mode de vie périurbain. Au-delà du centre du bourg structuré par une rue principale, quelques lotissements ou constructions individuelles récents se sont bâtis en périphérie mais restent en proximité de l'école et du centre du bourg. Le bourg connaît peu d'aménités et les déplacements sont dominés par la pratique automobile : $85,5 \%$ des actifs utilisent une voiture ou équivalent pour se rendre à leur travail. (INSEE)

15 Le quartier périurbain de l'étude est représenté par deux classes de CM1. L'école est intégrée à une commune de 26477 habitants en 2015 qui jouxte la grande métropole et qui a connu un développement périurbain important parce que située à proximité de deux grands bassins d'emplois. Le quartier de l'école appartient à un espace qui s'est développé depuis les années 1970 et est composé d'ensembles pavillonnaires et d'immeubles collectifs de petite taille dans un cadre de vie composé de commerces de proximité et d'espaces verts. Là encore, $82 \%$ des actifs travaillent dans une autre commune que celle de résidence d'après l'INSEE et $82,8 \%$ d'entre eux utilisent leur voiture pour se rendre sur leurs lieux de travail. 
Les trois autres écoles sont situées dans une grande métropole, dans trois quartiers très différents. Le premier, désigné "centre-ville » dans la typologie, est un quartier à proximité de l'hyper centre-ville situé dans la partie de la ville qui s'est développée au $\mathrm{XIX}^{\mathrm{e}}$ siècle. Le quartier est résidentiel et il est composé de maisons de maîtres et de petits immeubles collectifs dispersés. Il est pourvu d'un important réseau de transports en commun. La deuxième école de la métropole désignée comme "péricentre " est située dans un quartier qui s'est développé au $\mathrm{XIX}^{\mathrm{e}}$ et surtout au début du $\mathrm{XX}^{\mathrm{e}}$ siècle à partir d'avenues structurantes des anciens faubourgs maintenant totalement intégrés à la ville. L'habitat est donc mixte composé d'anciennes maisons d'artisans ou de petits fermiers rénovées, de maisons construites au long $\mathrm{du} \mathrm{XX}^{\mathrm{e}}$ siècle sur de petites parcelles et de grands ou petits collectifs construits à partir des années 1960. Les transports en commun sont accessibles et le quartier est plutôt résidentiel tout en proposant un grand nombre de commerces de proximité. La troisième école est située à la périphérie de la ville à proximité de deux autres communes et au-delà de la ceinture du périphérique. C'est un quartier structuré autour d'une avenue le long de laquelle on repère encore les fermes de cette ancienne zone maraîchère. Développé depuis peu, le quartier concentre des activités commerciales et industrielles avec quelques dépôts importants et des ensembles collectifs d'assez grande taille. Les commerces de proximité sont peu nombreux et dispersés, quelques équipements sportifs et de loisirs sont installés à proximité de l'école. Bien que l'étude concerne seulement trois quartiers de la métropole, on peut quand même relever, à titre de comparaison, les statistiques présentées dans les caractérisations précédentes. Ainsi, pour cette commune de 471945 habitants en 2015, les maisons individuelles représentent 16,5\% des habitats, et $73,5 \%$ des habitants travaillent dans la commune de résidence. Parmi les actifs, 53,5\% utilisent leur véhicule personnel pour se rendre au travail.

\section{Résultats : Les trajets domicile-école sont-ils des lieux d'exploration de l'autonomie?}

17 L'analyse statistique des questionnaires permet de constituer plusieurs catégories de résultats. La conscience de résider à proximité de l'école interroge l'habiter des élèves, au sens de leur capacité à éprouver la distance aux lieux, et peut, ainsi, être mise en relation avec la typologie des territoires. La question de l'autonomie des élèves et ainsi de leur capacité à construire des compétences spatiales sur cet espace-temps spécifique a été analysée de manière générale et en fonction des types de territoire. Les pratiques de mobilité seront mises en relation avec les moyens de déplacements et les temps de parcours.

\section{Quelle conscience les élèves ont-ils d'habiter le quartier de l'école ?}

La première question posée aux élèves était la suivante : "Est-ce que tu habites le quartier de l'école ?». Sur les 141 élèves de l'étude, $64 \%$ ont répondu favorablement à la question. Pour interpréter ce résultat, il faut prendre en compte plusieurs éléments. La notion d'habiter est là entendue dans le sens de résider. On peut faire une première hypothèse sur la perception du sens de «quartier ». Il semble que, pour la plupart d'entre eux, le quartier correspond à l'espace situé autour de l'école, parcouru à pied 
lors de la sortie effectuée pendant l'ingénierie didactique. Mais on peut également observer que les réponses sont différenciées par la situation des écoles (fig.2).

Figure 2 : Répartition des réponses de la conscience d'habiter le quartier de l'école en fonction de la typologie des territoires

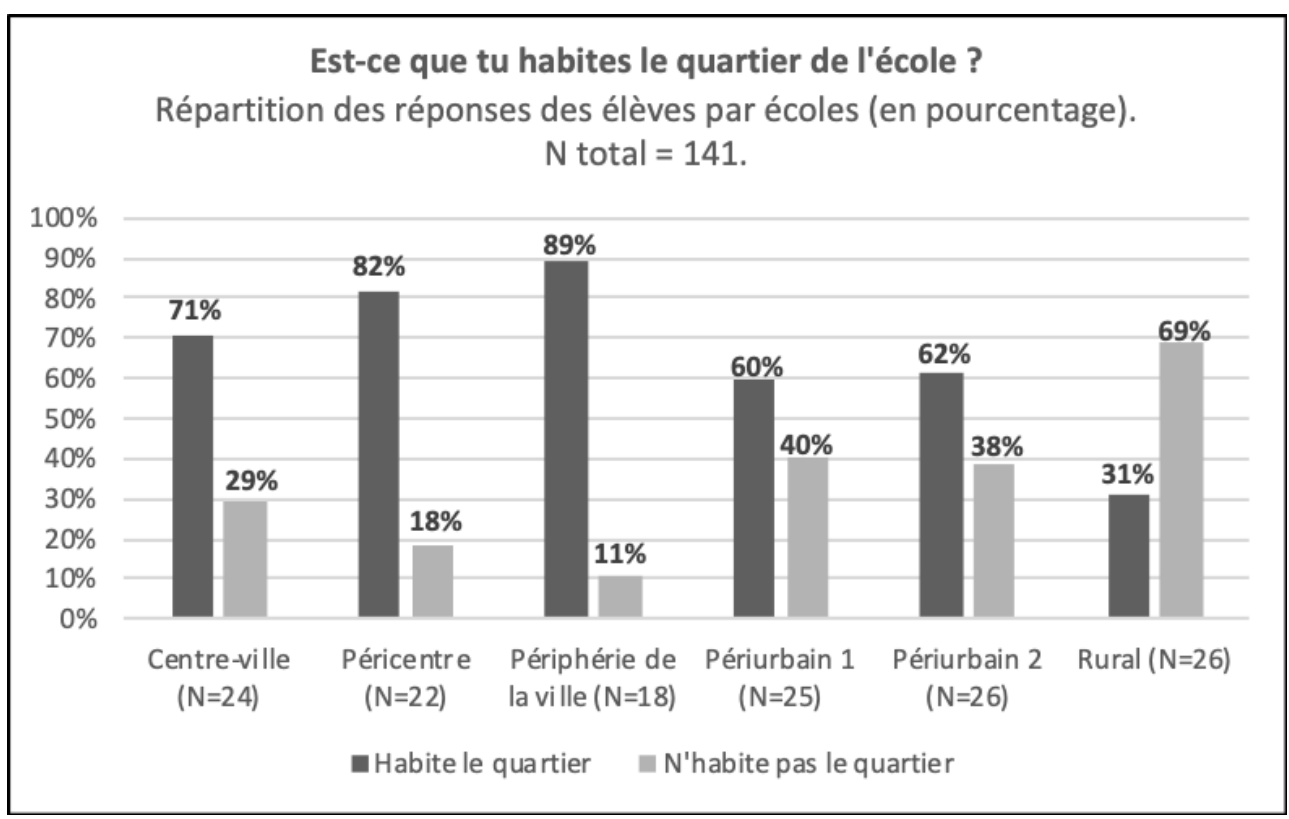

Ce graphique montre que la conscience d'habiter le quartier de l'école est plus importante dans les premières classes de la typologie, c'est-à-dire dans les quartiers urbains, tandis que pour les classes de l'école périurbaine et de l'école rurale, les élèves pensent habiter en dehors du quartier de l'école. C'est particulièrement marqué pour la classe rurale, puisque dans cette école $69 \%$ des élèves considèrent qu'ils n'y habitent pas. On peut s'étonner de ce résultat, car en termes de gestion scolaire, la majorité des élèves réside dans le quartier de l'école à laquelle ils sont rattachés. Ce qui nous conduit à une deuxième hypothèse qui suppose que les élèves ont répondu en mobilisant la perception de la distance entre leur domicile et leur école. Ainsi, dans les quartiers urbains, la densité de l'habitat permet certainement aux élèves de percevoir plus facilement la proximité entre leur domicile et leur école. A contrario, l'habitat plus étalé, typique des espaces périurbains et ruraux, augmente la perception de la distance, qui peut être réelle par ailleurs, et donne aux élèves un sentiment d'éloignement. Ainsi la physionomie et la structure des territoires étudiés semblent avoir une incidence sur la perception de la distance aux lieux chez les élèves. La représentation qu'ils se font de la distance entre leur lieu de résidence et leur lieu d'étude peut être considérée comme une manière de "faire avec l'espace ", et montre que les élèves ont déjà conscience qu'ils n'habitent pas de la même manière selon qu'ils vivent en milieu urbain, périurbain ou rural.

\section{Quelles expériences spatiales les élèves ont-ils sur le trajet domicile-école?}

Dans la deuxième partie du questionnaire, les élèves ont été interrogés sur leur manière d'effectuer le trajet entre leur domicile et leur école. Corroborant les travaux cités en amont, les élèves n'ont que très peu d'expérience autonome des trajets 
domicile-école, et ce quels que soient les territoires de la typologie. En effet, sur notre effectif de 141 élèves de 6 classes différentes, seulement $19 \%$ effectuent seuls le trajet domicile-école ; $81 \%$ des élèves sont accompagnés sur ce trajet. Sur les $81 \%$ des élèves qui viennent accompagnés à l'école, $99 \%$ le sont par un adulte. Un seul élève de l'étude vient à l'école accompagné d'un groupe d'enfants dont il est le plus âgé (Fig.3). Plusieurs facteurs permettent d'interpréter ce résultat.

Le facteur lié à l'âge des élèves n'est pas représentatif. En effet, les élèves interrogés sont âgés de 8 à 11 ans, et ils se répartissent dans des classes simples ou de double niveau. Quatre classes concernent uniquement des élèves de CM1 (9-10 ans) : au centreville, en périphérie et dans le quartier périurbain. La classe rurale concerne des élèves de CM1 et CM2 (9-11 ans) et celle située au péricentre est composée d'élèves de CE2 et $\mathrm{CM} 1$, c'est-à-dire en 8 et 10 ans. Au vu des résultats, il paraît difficile d'établir un lien entre l'âge et l'autonomie, les élèves les plus âgés (rural $=12 \%$ ) ayant moins d'autonomie que les élèves les plus jeunes (péricentre $=33 \%$ ).

Figure 3 : Autonomie des élèves sur le trajet domicile-école par classe. Les résultats sont exprimés en valeur relative $(N=141)$

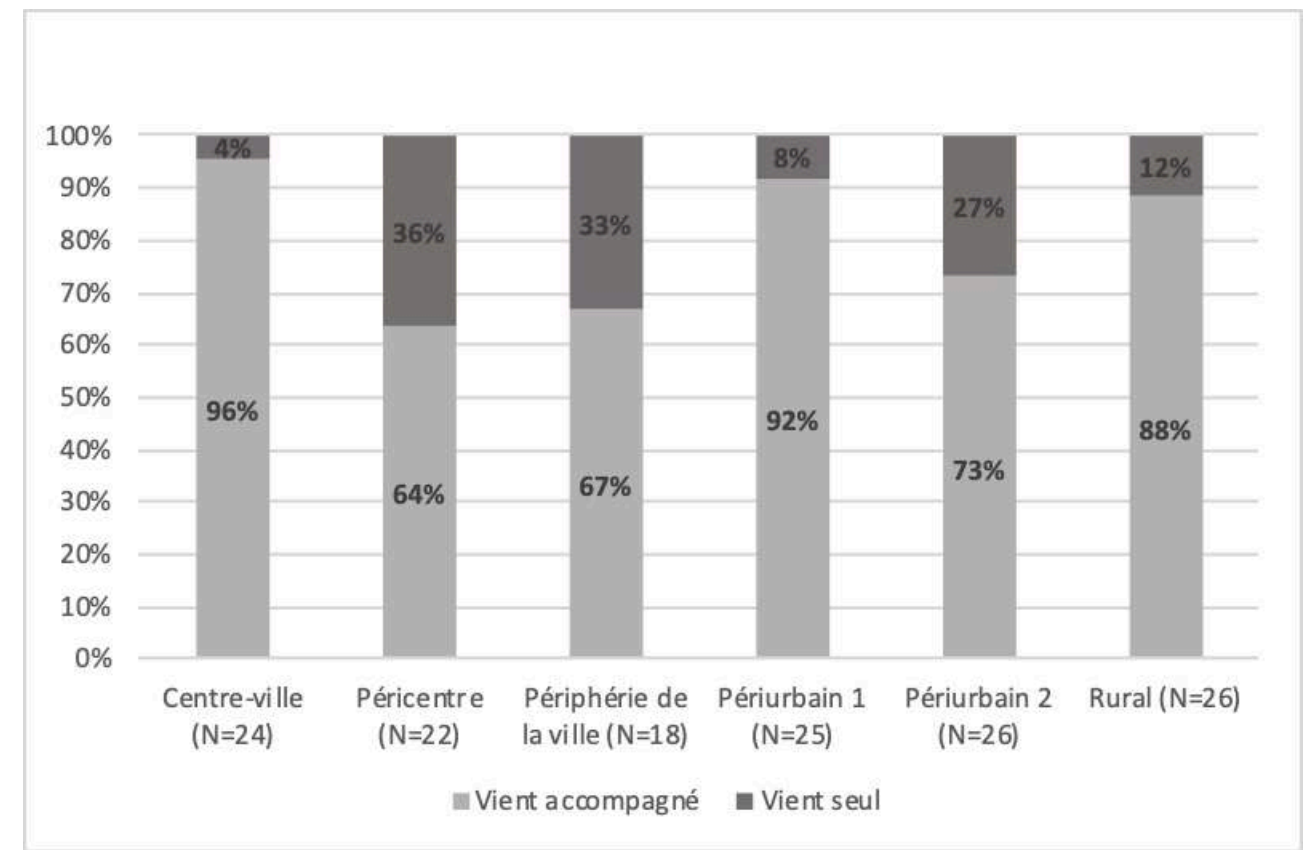

La longueur du trajet explique peut-être la faible autonomie des élèves. Pourtant $68 \%$ des élèves accompagnés le sont sur un trajet de moins de 5 minutes et $21 \%$ sur un trajet d'une dizaine de minutes (fig. 4). Même si ces données s'appuient sur la perception du temps de trajet par les élèves, ce qui peut être discuté du point de vue de leur capacité à estimer une durée, il ne semble pas y avoir d'explication nette entre la distance et l'autonomie. Tracy MacMillan (2005) mobilise le sentiment de sécurité (réel ou perçu) en tant que facteur d'explication de la faible autonomie des élèves. Pourtant, les trois écoles qui montrent les pratiques d'accompagnement les plus élevées relèvent de territoires très différents : le centre-ville ( $=96 \%)$, une école péri-urbaine ( $=92 \%)$, une école rurale $(=88 \%)$. Dans ces trois lieux, les conditions de sécurité, liée notamment à la circulation automobile, sont très différentes. Au centre-ville, la densité de la circulation augmente la perception d'insécurité, mais en territoire périurbain ou rural 
où les aménagements pour les cyclistes ou les piétons sont plus fréquents, les pratiques d'autonomie des élèves n'y sont pas plus importantes.

Figure 4 : Diagrammes circulaires présentant les temps et les moyens de transports utilisés par les élèves venant accompagnés à l'école

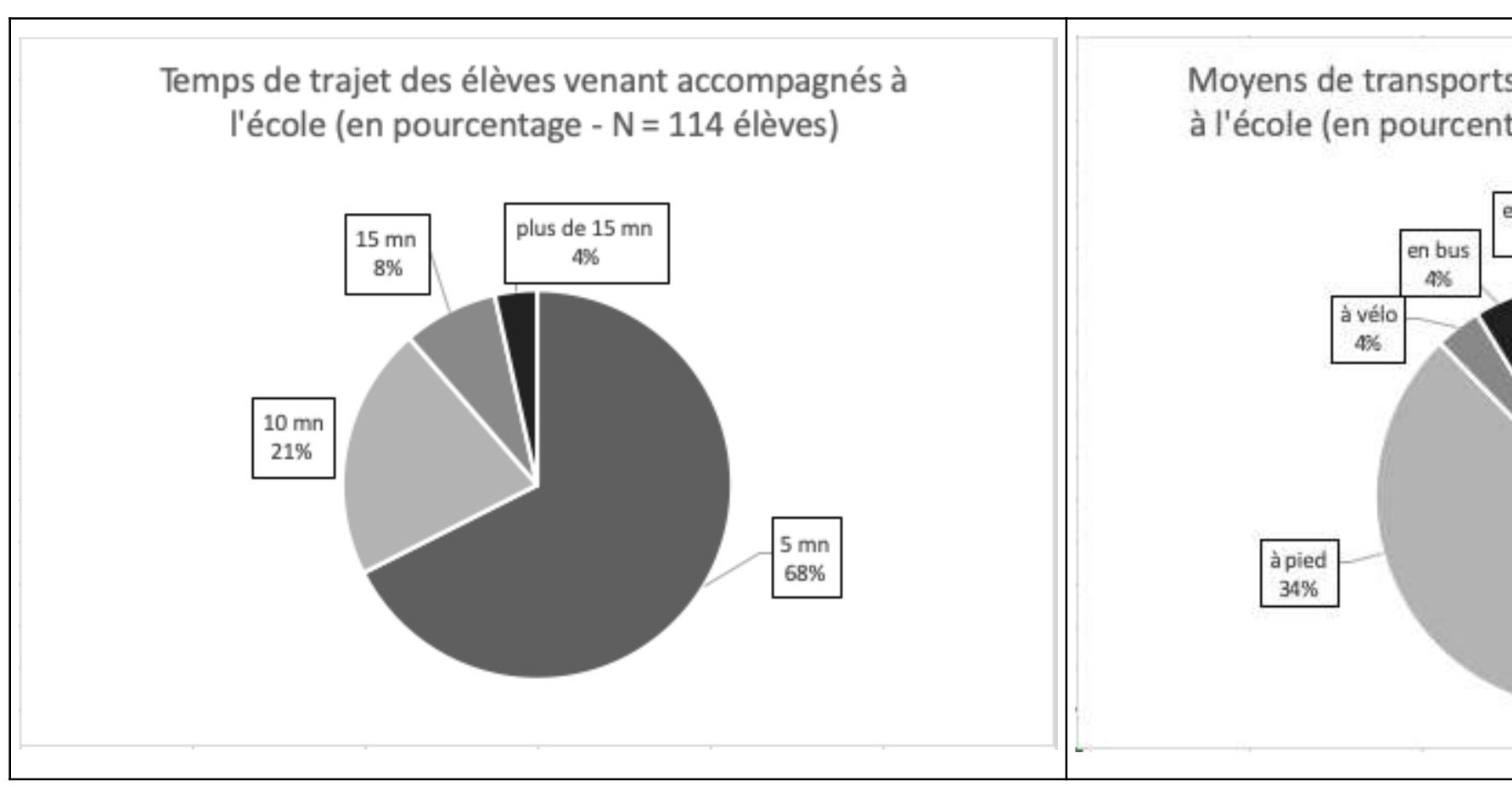

En s'appuyant sur les travaux de Paul Lewis et Juan Torres pour Montréal (2010), l'hypothèse que le facteur décisif de l'autonomie des élèves sur le trajet domicile-école serait lié aux pratiques de mobilité des parents paraît déterminante. La figure 5 montre une grande diversité de pratiques de mobilité selon les territoires. 
Figure 5 : Moyens de transports utilisés selon les écoles (pour les élèves accompagnés sur le trajet domicile-école). $\mathrm{N}=114$

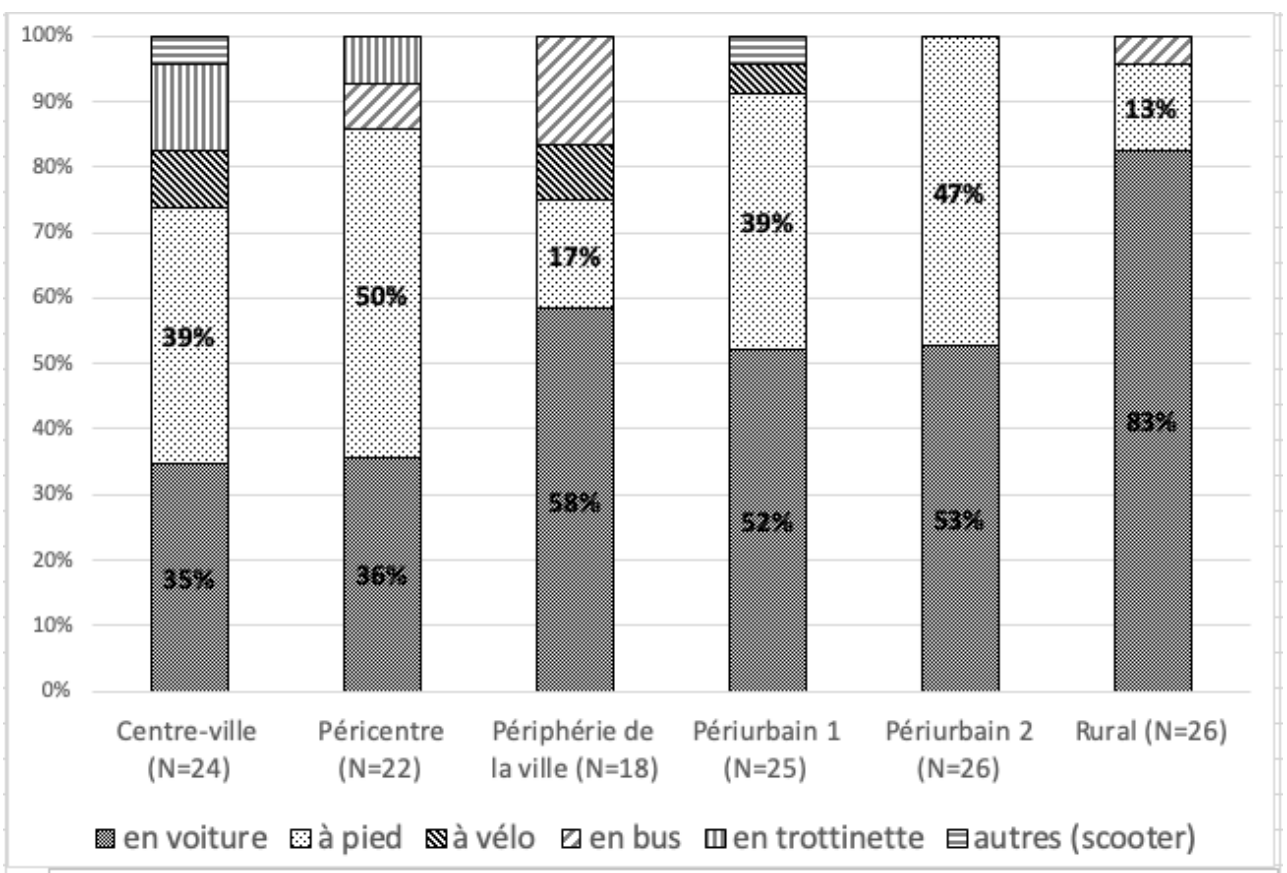

En effet, il y a une plus grande variété de moyens de transport dans les territoires urbains (centre-ville, péricentre, périphérie de la ville) que dans les territoires périurbains et ruraux (fig.5). C'est dans l'école dite "rurale» que le pourcentage d'élèves effectuant le trajet en voiture est le plus significatif : $83 \%$ des élèves. Et, à l'inverse, c'est dans les écoles du «centre-ville» et du "péricentre» que les pourcentages de trajet effectués en voiture sont les plus faibles, respectivement 35 et $36 \%$ des élèves. On voit ainsi apparaitre des contrastes entre les espaces centraux de la métropole, les espaces périphériques et périurbains et l'espace rural. Ainsi, il existe, dans les pratiques d'accompagnement des parents, un gradient progressif d'utilisation de la voiture en fonction de l'éloignement du centre de la métropole. Il a été largement documenté que l'habiter périurbain est caractérisé, entre autres, par l'hégémonie automobile et les pratiques de mobilités en boucles programmées (Cailly, 2008; Didier Fèvre, 2014; Jaillet, 2013). Le trajet domicile école dans les écoles périurbaines et rurales semble relever de ce type de parcours intégré au sein d'un circuit organisé en boucle autour du domicile. Dans l'école rurale, au mode de vie périurbain, $83 \%$ des élèves sont déposés à l'école en voiture, dans les écoles du quartier périurbain, c'est plus de la moitié des élèves, les autres sont accompagnés majoritairement à pied. En revanche, au centre-ville, les pratiques de mobilités sont plus diversifiées : la marche ( = $39 \%$ ) est supérieure à l'accompagnement motorisé ( $=35 \%)$, vélo, trottinette ou scooter représentant le dernier tiers (respectivement $=9 \%, 13 \%, 4 \%$ ).

On peut donc conclure de cette interprétation que le trajet domicile-école représente une forme de mobilité dont les enfants sont assez peu acteurs et qu'elle est liée avant tout aux pratiques de mobilité des familles. Par ailleurs, on a pu constater que dans les territoires ruraux et périurbains, les enfants ont une forme de mobilité davantage liée à la motorisation et donc peut-être moins de possibilité d'en tirer une expérience spatiale. 


\section{Discussion}

Ces résultats méritent d'être discutés. Tout d'abord, ce travail se limite à un échantillon de six écoles ce qui en relativise considérablement la portée. Par ailleurs il s'appuie sur des pratiques déclarées qui n'ont pas été vérifiées dans le cadre d'un croisement avec les données familiales (distance réelle à l'école, situation familiale etc...). Enfin, le questionnaire renseigne sur un aspect limité des pratiques de mobilité enfantine. Il serait intéressant de renseigner à la fois les autres formes de mobilité (jeux dans le quartier, visites aux amis, courses à proximité du domicile ou déplacement vers les activités de loisirs par exemple) qui permettraient d'interroger les manières d'habiter des élèves mais également la part d'autonomie dont ils seraient eux-mêmes demandeurs, afin de ne pas en supposer le besoin.

Il s'agit, alors, d'interroger le rôle de l'école et en particulier de l'enseignement de la géographie pour enrichir l'expérience spatiale des élèves. On peut se demander pourquoi et comment, au-delà du prescrit, l'école mobilise l'expérience spatiale des élèves? Il faut d'abord prendre conscience que les élèves n'ont pas nécessairement des pratiques spatiales quotidiennes importantes. Les questionnaires passés dans les classes et leurs résultats ont été reçus avec beaucoup d'étonnement par les enseignants qui imaginaient une plus grande autonomie de leurs élèves, notamment dans les classes périurbaines et rurales. Faire passer ce type de questionnaire en classe peut être le point de départ d'une recherche avec les élèves sur les pratiques de mobilité.

Mais il y a également nécessité à investir encore plus fortement les pratiques de sorties piétonnes de découverte de l'espace proche de l'école afin de permettre aux élèves de construire des compétences de spatialité. L'espace proche est un point de départ légitime pour construire un questionnement géographique sur les manières d'habiter. Lors de la mise en place de l'ingénierie didactique avec les six classes, la sortie dans le quartier a été un temps fort pour la plupart des élèves. Parce qu'elle permettait d'éprouver l'itinéraire qui avait été construit, de trouver des indices pour répondre aux questions qui formaient le canevas de l'enquête mais aussi parce qu'elle était l'occasion de montrer ce que les élèves savaient sur leur environnement proche, la sortie et son exploitation ont été d'importants moments d'apprentissage. Pendant les entretiens passés auprès de quelques élèves de chaque classe ${ }^{8}$, c'est souvent la sortie qui a été citée comme le moment préféré de l'enquête géographique menée en classe. Plusieurs élèves ont expliqué qu'ils n'avaient jamais réalisé de sortie dans l'environnement proche, en géographie, dans leur cursus scolaire et, pour ceux qui en avaient déjà réalisé, celles-ci s'étaient limitées aux abords immédiats de l'école. Ils ont évoqué plusieurs raisons pour justifier leur intérêt pour cette pratique : la sortie a bien évidemment été l'occasion de "faire du sport», «de prendre l'air », mais aussi "d'apprendre à se repérer ", " de repérer par où on est passé ", et surtout la possibilité de découvrir des lieux qu'ils ne connaissaient pas. L'un évoque la découverte du city-stade à quelques centaines de mètres de son école et il explique «Maintenant j'y vais avec (il cite trois prénoms d'enfants de sa classe). On en a parlé à nos parents pour qu'ils nous y amènent. "; l'autre évoque cette rue à proximité de la forêt et à quelques mètres de son chemin habituel par laquelle il n'était jamais passé. Enfin une autre élève explique que c'est essentiel «pour ceux qui ne savent pas, comme (elle cite deux prénoms d'enfants de la classe nouvellement arrivés), c'est plus intéressant parce que ça leur apprend comment c'est (elle cite le nom de la commune). » Une élève d'une autre classe, arrivée dans l'année, explique également 
l'intérêt de la sortie en termes d'intégration " pour connaître les mêmes choses que les autres ».

\section{Conclusion}

Ainsi, le champ des mobilités enfantines est particulièrement intéressant pour la didactique de la géographie car il permet de prendre appui sur l'expérience spatiale des élèves pour les aider à construire des compétences de spatialité. Le nouveau programme de géographie de cycle 3 insiste largement sur l'intérêt de s'appuyer sur l'espace vécu des élèves, en proposant de faire partir la plupart des questionnements géographiques des expériences d'habitation des élèves, parce que, comme l'explique Michel Lussault, « l'« Habiter » (...) mobilise à la fois l'expérience immédiate et médiate de l'individu (...) pour essayer de lui faire comprendre ce qu'on peut constituer comme apprentissage de lui-même, de sa relation aux autres et du monde, à partir de cette expérience » (Lussault, 2018, p. 17). Le trajet domicile-école est une expérience spatiale pertinente, car vécue quotidiennement par les élèves et donc l'occasion d'en repérer les lieux, d'en éprouver la distance et d'y faire les premières observations. Une grande part des apprentissages spatiaux ne peut se faire sans l'expérience directe du «faire avec » l'espace et l'école primaire a ici une opportunité réelle d'action pour travailler la spatialité des élèves. Comme le dit une des élèves de l'étude : «j'ai aimé imaginer le chemin sur le plan et puis sortir après, parce que ça m’apprend à découvrir des lieux ».

\section{BIBLIOGRAPHIE}

BAILLY A. S., 1977, La Perception de l'espace urbain : Les concepts, les méthodes d'étude, leur utilisation dans la recherche urbanistique, Paris, Ed. Centre de recherche d'urbanisme, $264 \mathrm{p}$.

BAILLY A.-S., 1989, L'imaginaire spatial. Plaidoyer pour la géographie des représentations, Espace, Temps, vol. 40, $\mathrm{n}^{\circ} 1$, p. 53-58.

DEPEAU S., 2013, Mobilité des enfants et des jeunes sous conditions d'immobilité ? Ce que circuler signifie, E-Migrinter, 1 septembre, $\mathrm{n}^{\circ}$ 11, p. 103-115.

DEPEAU S., RAMADIER T., 2005, Les Trajets Domicile-École en Milieux Urbains : Quelles conditions pour l'autonomie de l'enfant de 10-12 ans ? Psychologie et Société, vol. 8, p. 81-112.

DEVAUX J., OPPENCHAIM N., PROULHAC L., 2016, L'évolution des pratiques de mobilité des adolescents depuis 20 ans en Île-de-France : quelle influence des variables sociales et territoriales sur les inégalités de genre ? Métropoles, 15 juin, $\mathrm{n}^{\circ} 18$.

DIDIER FÈVRE C., 2014, Occuper les entre-deux : les pratiques spatiales de lycéens à Sens et à LaQueue-Lez-Yvelines, Carnets de Géographes, 11 décembre, $\mathrm{n}^{\circ} 7$.

FOTEL T., THOMSEN T. U., 2003, The Surveillance of Children's Mobility, Surveillance and Society, vol. $1, \mathrm{n}^{\circ} 4$.

FRÉMONT A., 1976, La région, espace vécu, Paris, PUF, 234 p. 
GASTER S., 1991, Urban Children's Access to their Neighborhood: Changes Over Three Generations, Environmental Behaviour, 1 janvier 1991, vol. 23, nº 1, p. 70-85.

GAUJAL S., 2016, Une géographie à l'école par la pratique artistique, Thèse de doctorat, Université Paris 7 - Denis Diderot.

HERTIG P., 2009, Didactique de la géographie et formation initiale des enseignants spécialistes, Lausanne, UNIL - Université de Lausanne, Institut de géographie.

HUGUENIN-RICHARD F., 2010, La mobilité des enfants à l'épreuve de la rue : Impacts de l'aménagement de zones 30 sur leurs comportements, Enfances, Familles, Générations, n 12 , p. 66-87.

KAUFMANN V., WIDMER É. D., 2005, L'acquisition de la motilité au sein des familles, Espaces et Sociétés, vol. n 120-121, n 1, p. 199-217.

KOLB D. A., 2014, Experiential Learning: Experience as the Source of Learning and Development, FT Press, $417 \mathrm{p}$.

LAZZAROTTI O., 2014, Habiter le monde, Paris, La Documentation Photographique., $\mathrm{n}^{\circ}$ 8100, 63 p.

LEININGER-FREZAL C., 2018, Training Primary teachers through experiential geography, European Journal of Geography, vol. 9, n 2, p. 17.

LEWIS P., TORRES J., 2010, Les parents et les déplacements entre la maison et l'école primaire : quelle place pour l'enfant dans la ville? Enfances Familles Générations, ${ }^{\circ}$ 12, p. 44.

LUSSAULT M., 2013, Spatialité, in LEVY J. (dir.), Dictionnaire de la Géographie, de l'Espace et des Sociétés, Paris, Belin, p. 947-950.

LUSSAULT M., 2015, L'expérience de l'habitation, Annales de Géographie, 15 septembre, nº 704, p. 406-423.

LUSSAULT M., 2013, Compétences de spatialité, in LEVY J. (dir.), Dictionnaire de la Géographie, de l'Espace et des Sociétés, Paris, Belin, p. 212-214.

LUSSAULT M., 2015, Entretien : «L'école ne peut pas être indifférente à l'expérience singulière du monde ", Diversité, vol. 179, n Habiter l'école. Lieu ouvert, lieu fermé ? p. 7-10.

LUSSAULT M., 2018, " Mettre l'expérience extrascolaire en lien avec la pratique scolaire ", Diversité, 2018, vol. L'expérience du territoire. Apprendre dans une société durable, n 191, p. 5.

MCMILLAN T. E., 2005, Urban Form and a Child's Trip to School: The Current Literature and a Framework for Future Research, Journal of Planning Literature, mai, vol. 19, n 4, p. 440-456.

RAMADIER T., 2007, Mobilité quotidienne et attachement au quartier : une question de position? in AUTHIER J-Y., BACQUE M-H., GUERIN-PACE F. (dir.), Le quartier : Enjeux scientifiques, actions politiques et pratiques sociales, Paris, La Découverte, collection « Recherche », Chapitre 10, p. 127-138.

RETAILLÉ D., 1997, Le monde du géographe, Paris, Presses de Sciences Po, 283 p.

STOCK M., 2015, Habiter comme « faire avec l'espace ». Réflexions à partir des théories de la pratique, Annales de Géographie, 15 septembre, $\mathrm{n}^{\circ}$ 704, p. 424-441.

THÉMINES J.-F., 2007, Enseigner la géographie : un métier qui s'apprend (Ressources formation : enjeux du système éducatif), Paris, Hachette, 158 p.

VERGNOLLE MAINAR C., GAUJAL S., LEININGER-FRÉZAL C., 2017, La géographie scolaire et le territoire de proximité, in BARTHES A., CHAMPOLLION P., ALPE Y. (dir.), Permanences et 
évolutions des relations complexes entre éducation et territoires, ISTE Editions, série Education, p. 139-154.

\section{NOTES}

1. La recherche en cours vise la construction et l'évaluation d'une ingénierie didactique sur l'enseignement géographique à partir de l'environnement proche des élèves en classe de CM1. Une des modalités d'évaluation des compétences de spatialité des élèves s'appuie sur l'analyse de dessin de représentation de l'environnement proche avant et après l'ingénierie. Le recueil de données avait pour but de lever un biais d'interprétation en écartant toute influence de pratique autonome de l'environnement proche que les élèves auraient pu développer à leur entrée en CM1 en pratiquant seuls le trajet entre leur domicile et leur école.

2. Programme de géographie de cycle 3. BOEN spécial n 11 du 26 novembre 2015 (consolidé dans le BOEN $n^{\circ} 30$ du 26 juillet 2018) - consulté le 26/04/2019 http:// cache.media.eduscol.education.fr/file/programmes_2018/20/2/

Cycle_3_programme_consolide_1038202.pdf

3. Document d'accompagnement du programme 2015, publié en mars 2016. http:// eduscol.education.fr/cid98981/s-approprier-les-differents-themes-programme.html

4. Des dessins de l'espace autour de l'école ont été collectés auprès de tous les élèves de l'étude avant et après l'ingénierie didactique. Ces dessins sont en cours de traitement et d'analyse mais ils révèlent d'importants progrès dans les points de vue, la schématisation, la situation des éléments, et l'échelle employée.

5. Dossier complet commune sur le site de l'INSEE: https://www.insee.fr/fr/information/ 3544265

6. Géoportail : https://www.geoportail.gouv.fr

7. Les données présentées ici, proviennent des Dossiers communes de l'INSEE : - Commune «rural» Insee, https://www.insee.fr/fr/statistiques/2011101?geo=COM-31349 - Commune "périurbain » Insee, https://www.insee.fr/fr/statistiques/2011101?geo=COM-31557 - Métropole Insee, https://www.insee.fr/fr/statistiques/2011101?geo=COM-31555

8. Ces propos d'élèves sont présentés à titre illustratifs. Ils sont issus d'un ensemble de 52 entretiens passés avec quelques élèves de chaque classe. Le but de ces entretiens est de permettre aux élèves d'expliquer les dessins de représentation de l'espace qu'ils ont réalisés avant et après l'ingénierie didactique et ces entretiens sont en cours de traitement selon une méthodologie propre à ce champ d'étude. Dans le cadre de l'entretien, il leur a également été demandé de faire un retour sur les différentes étapes du travail réalisé dont la sortie. Les propos cités appartiennent à cette partie de l'entretien et les principales raisons utilisées par les élèves pour justifier l'importance donnée à la sortie sont présentées.

\section{RÉSUMÉS}

Les nouveaux programmes français de géographie du cycle 3 centrent les apprentissages spatiaux sur les expériences directes des élèves autour de la notion d'habiter. Les trajets domicile-école peuvent ainsi représenter un terrain d'étude pertinent pour révéler des expériences spatiales 
vécues à la fois singulièrement, collectivement et quotidiennement. L'étude des pratiques de mobilités enfantines sur ce type de trajet pour des élèves de CM1 de six classes dans des territoires différents peut permettre de révéler la réalité de ces expériences spatiales, en termes d'autonomie, de modes et de temps de déplacements. Les résultats permettent de questionner les conditions de possibilité pour transformer ces expériences spatiales en apprentissages géographiques.

The new geography programmes in upper primary years focus spatial learning on childrens" direct experiences around the notion of living environment. Home-school commutes can thus represent a relevant field of study to reveal spatial experiences that are lived both singularly, collectively and daily. The study of child mobility on this type of journey for 9-10 year-old children from six different classes and in different territories can reveal the reality of these spatial experiences, in terms of autonomy, modes and travel time. The results allow us to question the conditions under which it is possible to transform these spatial experiences into geographical learning.

\section{INDEX}

Mots-clés : mobilité enfantine, habiter, trajet domicile-école

Keywords : child mobility, living environment, home-school commute

\section{AUTEUR}

\section{ELSA FILÂTRE}

Doctorante et formatrice à l'INSPE de Toulouse Occitanie-Pyrénées, Laboratoire GEODE UMR 5602 CNRS Université Toulouse Jean-Jaurès, 5 allées Antonio Machado, 31058 Toulouse Cedex

Elsa.filatre@univ-tlse2.fr 